\title{
miR-101 Inhibiting Cell Proliferation, Migration and Invasion in Hepatocellular Carcinoma through Downregulating Girdin
}

\author{
Ke Cao ${ }^{1,4}$, Jingjing Lif,, Yong Zhao', Qi Wang', Qinghai Zeng ${ }^{3}$, Siqi He', Li Yu', Jianda Zhoư ${ }^{3}$, and \\ Peiguo Cao, ${ }^{1, *}$
}

\begin{abstract}
miR-101 is considered to play an important role in hepatocellular carcinoma (HCC), but the underlying molecular mechanism remains to be elucidated. Here, we aimed to confirm whether Girdin is a target gene of miR-101 and determine the tumor suppressor of miR-101 through Girdin pathway. In our previous studies, we firstly found Girdin protein was overexpressed in HCC tissues, and it closely correlated to tumor size, $T$ stage, TNM stage and Edmondson-Steiner stage of HCC patients. After specific small interfering RNA of Girdin was transfected into HepG2 and Huh7.5.1 cells, the proliferation and invasion ability of tumor cells were significantly inhibited. In this study, we further explored the detailed molecular mechanism of Girdin in HCC. Interestingly, we found that miR-101 significantly low-expressed in HCC tissues compared with that in matched normal tissues while Girdin had a relative higher expression, and miR-101 was inversely correlated with Girdin expression. In addition, after miR-101 transfection, the proliferation, migration and invasion abilities of HepG2 cells were weakened. Furthermore, we confirmed that Girdin is a direct target gene of miR-101. Finally we confirmed Talen-mediated Girdin knockout markedly suppressed cell proliferation, migration and invasion in HCC while downregulation of miR-101 significantly restored the inhibitory effect. Our findings suggested that miR-101/Girdin axis could be a potential application of HCC treatment.
\end{abstract}

\section{INTRODUCTION}

According to 2012 American cancer statistics, hepatocellular carcinoma ( $\mathrm{HCC})$ is the fifth most common malignant tumor

\footnotetext{
${ }^{1}$ Department of Oncology, Third Xiangya Hospital, Central South University, ${ }^{2}$ Department of Pathology, Xiangya Basic Medical College, Central South University, ${ }^{3}$ Department of Plastic Surgery, Third Xiangya Hospital, Central South University, Changsha, Hunan, China, ${ }^{4}$ These authors contributed equally to this work.

*Correspondence: csucaopeiguo@163.com
}

Received 3 June, 2015; revised 7 September, 2015; accepted 14 October, 2015; published online 7 January 2016

Keywords: Girdin, invasion and metastasis, miR-101, primary hepatocellular carcinoma cell, proliferation with an increasing incidence (Siegel et al., 2013). HCC is difficult to diagnose as early-stage disease, resulting in poor survival. In recent years, great advances have been made in diagnostic techniques and treatment of HCC, yet the overall survival of patients is still relatively low (Tameda et al., 2014). Therefore, the molecular mechanisms underlying HCC is expecting for further research.

MicroRNAs are conserved non-coding RNAs with the length of 19-25nt, which regulate gene expression at the posttranscriptional level (Kim et al., 2015; Peng et al., 2015; Xu et al., 2013). The abnormal miRNA expression has been reported in many kinds of cancer, whereby miRNA plays act as either tumor suppressor or promoter (Landgraf et al., 2007). Recent studies have revealed that a series of miRNAs are played crucial roles in HCC progression. MiR-195 has been proved to function as a tumor suppressor by inhibiting CBX4 in HCC (Zheng et al., 2015). However, miR-21 acts as promoter for tumor metastasis in HCC (Zhou et al., 2013). These miRNAs can be used as diagnostic markers or therapeutic targets for HCC. The above research findings highlight the importance of miRNAs in the oncobiological behavior, but their specific molecular mechanisms remain to be elucidated.

Girdin protein was discovered in 2005 and according to its functions was named as girders of actin filaments, Akt phosphorylated enhancer, Hook-related protein 1 and transport vesicle-related $\mathrm{G} \alpha$ binding protein, respectively (Anai et al., 2005; Enomoto et al., 2005; Le-Niculescu et al., 2005; Simpson et al., 2005). Human Girdin protein was encoded through coiled-coil domain containing $88 \mathrm{~A}(\mathrm{CCDC} 88 \mathrm{~A})$ gene that is located on chromosome 2p16.1. Numerous studies have shown that Girdin is highly expressed in many styles of tumor tissues, including gastric cancer (Wang et al., 2014), esophageal cancer (Shibata et al., 2013), colorectal cancer (Zhang et al., 2014) and breast cancer (Jin et al., 2013). In our previous researches, high expression level of Girdin was found in HCC tissues and it closely correlated to tumor size, T stage, TNM stage and Edmondson-Steiner stage of HCC patients. After specific small interfering RNA of Girdin was transfected into HepG2 and Huh7.5.1 cells, the proliferation and invasion ability of tumor cells were significantly inhibited (Cao et al., 2015). However, the concrete mechanisms of Girdin on proliferation, migration and invasion in HCC remain to be elucidated.

According to multiple microRNA target gene prediction software packages, such as TargetScan and miRanda, Girdin mRNA 
3'-UTR is highly predicted to be a target gene of miR-101. Based on these findings, we have proposed the hypothesis that miR101 inhibits HCC cell proliferation, migration and invasion by directly regulating Girdin.

In this study, we found that miR-101 expression in the HCC tissue was significantly downregulated compared with that in the para-cancer tissue while Girdin mRNA expression in HCC tissue was upregulatied compared with that in the para-cancer tissue. Upon miR-101 transfection, the proliferation, migration and invasion abilities of HepG2 cells were weakened. In addition, the dual luciferase assay confirmed that Girdin is the direct target gene of miR-101. Finally we confirmed Talen-mediated Girdin knockout remarkably suppressed cell proliferation, migration and invasion abilities in HCC while down-regulation of miR-101 significantly restored the inhibitory effect. Thus, miR101/Girdin axis may be a promising molecular target for HCC therapy.

\section{MATERIALS AND METHODS}

\section{Tissue samples and cell lines}

Forty-five cases of specimens of HCCs and the para-cancer tissues (the distance between the tissue and cancer tissue was $\geq 2 \mathrm{~cm}$ ) were selected from Xiangya Hospital and the Third Xiangya Hospital of Central South University. All patients had signed informed consent forms and the study had received approval from Xiangya Hospital Ethics Committee. Human hepatocellular carcinoma cell strain HepG2 (ATCC, USA) was purchased from Cell Biology Laboratory, Xiangya School of Medicine, Central South University. HepG2 cells were cultured in a medium containing DMEM and $10 \% \mathrm{FBS}$ in a $5 \% \mathrm{CO}_{2}$ incubator at $37^{\circ} \mathrm{C}$.

\section{qRT-PCR}

The Girdin amplification primer sequence was downloaded from NCBI and Primer5.0 software was used to design primers. The primer sequences of Girdin were as follows: forward 5'GACCAACTAGAGGGAACTCG-3' and reverse 5'-TACTTTGT TTCTGTGCCATT-3'. The primer sequences of $\beta$-actin sense strand was: AGGGGCCGGACTCGTCATACT and the antisense strand was: GGCGGCACCACCA TGTACCCT. Both miR-101 (HmiRQP0020) and U6 (HmiRQP9001) primers were purchased from Guangzhou Fulengen Co., Ltd. (China). TRIzol method (Invitrogen) was used to extract the total RNA of cells and tissues. The purity and integrity of RNA was examined on a Micro-UV Spectrophotometer and RNA electrophoresis. Total RNAs was reverse- transcribed into cDNA with miScript II RT Kit (Qiagen, USA) on an ABI-9600 PCR Amplifier (PerkinElmer) detected by ABI PRISM 7700 Sequence Detection System (PerkinElmer). Samples were compared by using the relative expression level was calculated via the $2^{-\triangle \triangle C T}$ method. Girdin mRNA expression level was normalized to $\beta$-actin, while miR101 expression level was normalized to U6.

\section{Cell transfection}

miR-101 mimics, miR-101 inhibitor and miRNA NC mimics were purchased from Guangzhou Fulengen Co., Ltd. (China). Talen kit was purchased from SiDanSai Company (China). The HepG2 cells were inoculated into the $60 \mathrm{~mm}$ cell culture dish $(5$ $\mathrm{ml} /$ well) by density of $1 \times 10^{5} \mathrm{cell} / \mathrm{s} / \mathrm{ml}$. When the cells reached $80 \%$ confluence, Lipofectamin2000 (Invitrogen) was used to transfect miR-101 mimics, miR-101 inhibitor and miRNA NC mimics into HepG2 cells according to the instructions, respectively. According to the method reported by literatures, plasmids were transfected into HepG2 cells in order to perform the knockout of the Girdin gene (Cao et al., 2014). 48h after transfection, the fluorescence microscopy and the qRT-PCR were used to detect whether the cell cloning process was successful.

\section{CCK-8 test}

$48 \mathrm{~h}$ after transfection, the cells were inoculated to a 96-well plate by $5 \times 10^{3}$ cells for each well. Five repeated wells were set in each group. Cells were cultured for $24,48,72 \mathrm{~h}$, respectively, and CCK-8 was added to each well for $2 \mathrm{~h}$ at $37^{\circ} \mathrm{C}$. ELIASA was used to detect absorbency value at a wavelength of $450 \mathrm{~nm}$.

\section{Scratch test and Transwell test}

The $48 \mathrm{~h}$ after transfection, the cells were inoculated to a 24well plate by $1 \times 10^{5}$ cells per well. When the cells covered the well bottom, the $10 \mu \mathrm{L}$ tip was used to scratch the cell monolayer. And a serum-free medium was used to wash it twice. Images were captured at different time points $(0$ and $24 \mathrm{~h})$ by microscopy to assess the rate of gap closure. Scratch healing rate $=($ scratch width at 0 time point - scratch width at 24 time points)/scratch width at hour 0 time point $\times 100 \%$.

The Transwell chamber was placed in a $24-$ well culture plate. A $500 \mu \mathrm{l}$ preheated serum-free medium was added into the invasion chamber. After transfection, the density of HepG2 cells was adjusted to $5 \times 10^{5}$ cells $/ \mathrm{ml}$ with a serum-free medium. A $100 \mu \mathrm{l}$ cell suspension was added into each upper chamber, and a $500 \mu \mathrm{l}$ medium containing $10 \%$ FBS was added into the lower chamber. After $24 \mathrm{~h}$, cotton swabs were used to wipe cells on the upper surface, while cells attached to the membranes were fixed in $4 \%$ paraformaldehyde for $20 \mathrm{~min}$ and stained with hematoxylin. The results of the Transwell assay were imaged and the number of invasive cells was evaluated by the OD value.

\section{Western blot}

The $48 \mathrm{~h}$ after transfection, the cellular protein was extracted. After the protein concentration was measured via the BCA method, $5 \%$ stacking gel and $12 \%$ separating gel were performed SDS-PAGE electrophoreses. After that, the substances were transferred to a PVDF membrane. Rabbit anti-human Girdin polyclonal primary antibody (SC-133371, Santa Cruz Biotechnology) was added and kept in a shaker at $4^{\circ} \mathrm{C}$ overnight, and goat anti-rabbit secondary antibody (111-035-003, Jackson) was added for incubation at $37^{\circ} \mathrm{C}$ for $1 \mathrm{~h}$. X-ray film was exposed, developed and fixed. The incubation and coloring of $\beta$ actin internal reference antibody were the same as the above mentioned methods, and the primary antibody and secondary antibodies used were mouse anti-human $\beta$-actin monoclonal antibody (BM0272, ABZOOM) and goat anti-mouse secondary antibody (111-035-008, Jackson), respectively.

\section{Dual luciferase assay}

psiCHECKTM-2 vector (Promega) was purchased from Auragene Bioscience Corporation Inc. (China). miR-101 binds to two sites of Girdin-3'UTR, the vectors of the two sites were built, respectively, which were named UTR-1 and UTR-2. Two restriction endonucleases, $\mathrm{Xhol}$ and Notl, were used to clone the target gene fragment into the vectors. The primer sequences of Girdin-3'UTR and mutant Girdin-3'UTR are shown below. When the confluence of cells reached $50-60 \%$ in the 24 -well plate, a $0.5 \mu \mathrm{g}$ reporter gene plasmid and miR-101 mimics and miRNA NC mimics were co-transfected into HepG2 cells. The $48 \mathrm{~h}$ after transfection, the Dual-Luciferase Reporter Assay System E1910 (Promega, USA) was used to detect the activities of firefly luciferase and renilla luciferase in each group, and ratio of 
$\boldsymbol{A}$

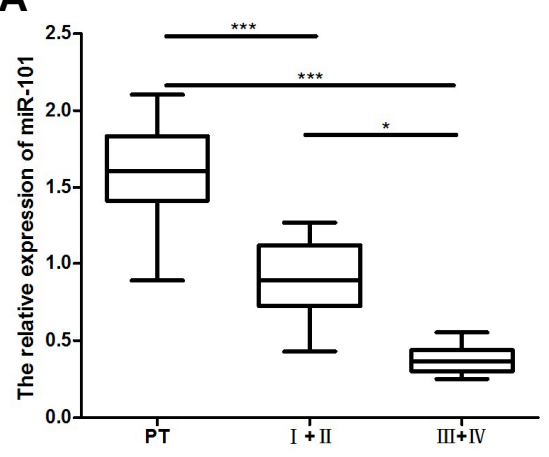

B

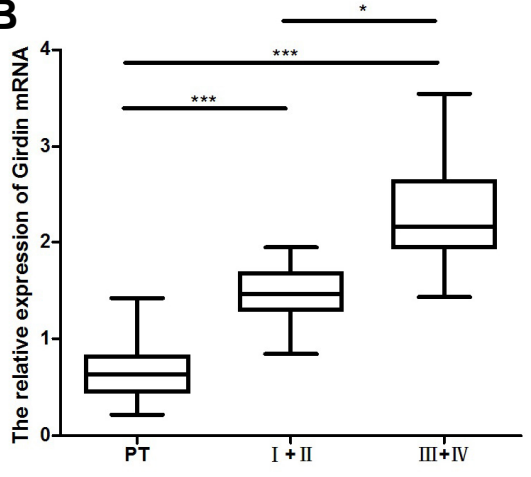

C

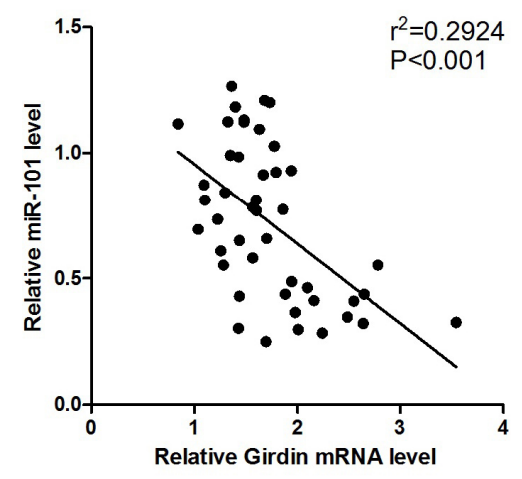

Fig. 1. miR-101 and Girdin expression in HCC tissue. (A) miR-101 expression level in HCC tissue was significantly lower than that seen in the para-cancer tissue, and miR-101 expression level in phase III/IV was lower than that seen in stage I/II. (B) Girdin expression level in HCC tissue was significantly higher than that seen in the para-cancer tissue, and Girdin expression level in stage III/IV was higher than that seen in stage I/II. PT: para-cancer tissue. Box-plot diagrams with median, maximum, minimum values and non-outlier range. (C) The correlation of miR-101 expression and Girdin mRNA level was analyzed. ${ }^{*} P<0.05,{ }^{* * *} P<0.001$.

renilla luciferase activity/firefly luciferase activity (R/F) was used as the relative activity value.

Girdin-3'-UTR gene sequence was downloaded from NCBI. Primer 5.0 software was used for primer design, and primer sequences are as follows:

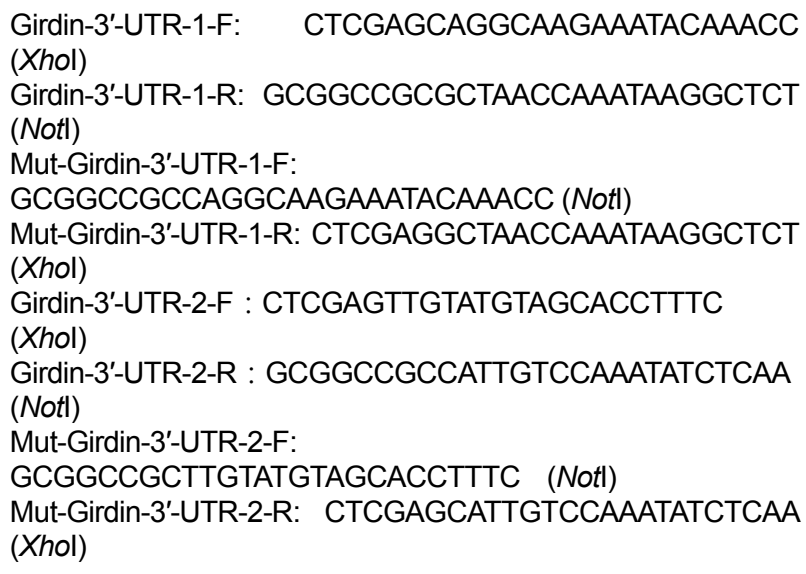

\section{Statistical method}

All tests were repeated at least three times, and SPSS 17.1 (USA) was used for statistical analysis. The experimental data were expressed as $X \pm S D(n=3)$, independent sample t-test was used for comparison between the two sets of data, and univariate variance analysis or rank sum test were used for comparison between groups. The difference of $P<0.05$ was statistically significant.

\section{RESULTS}

Low miR-101 expression and high Girdin expression in HCC tissue

In order to verify the abnormal expression of miR-101 in HCC, qRT-PCR method was used to detect miR-101 expression in 45 cases of HCC tissues and the para-cancer tissues. The experimental results showed that the miR-101 expression level in HCC tissue was significantly lower than that in the para- cancer tissue (Fig. 1A), and the miR-101 level in HCC tissue specimens in stage III/IV (15 cases) was lower than that found in stage $\mathrm{I} / \mathrm{II}$ (30 cases).

The result of Girdin mRNA expression in 45 cases of HCC tissues and the para-cancer tissues detected via RT-PCR method was consistent with previous results of our team. As shown in Fig. 1B, Girdin mRNA expression level in HCC tissue was significantly higher than that in the para-cancer tissue, and Girdin mRNA level in HCC tissue specimens in stage III/IV (15 cases) was also higher than that found in stage $1 / l l$ (30 cases).

The correlation analysis (Fig. 1C) of Girdin and miR-101 in HCC tissue confirmed the negative relationship between the miR-101 and Girdin which indicated that miR-101 may inhibited Girdin in HCC tissue.

In order to confirm whether miR-101 directly regulates Girdin, we transfected miR-101 mimics and miRNA NC mimics into HepG2 cells, respectively. The fluorescence microscopy (Fig. $2 \mathrm{~A}$ ) and RT-PCR method (Fig. 2B) were used to detect posttransfection miR-101 expression levels in HepG2 group (miR101-HepG2), blank group (NC-HepG2) and the negative control group (HepG2). The results showed that miR-101 had been successfully transfected into HepG2 cells.

\section{MiR-101 overexpression inhibits HepG2 cell proliferation ability}

CCK-8 method (Fig. 2C) was used to detect the effect of miR101 transfection on HepG2 cell proliferation. At 24, 48, $72 \mathrm{~h}$ time points, the proliferation rates in HepG2 group (miR-101HepG2) receiving miR-101 transfection were significantly lower than that in the group (NC-HepG2) and the untreated group (HepG2), while there was no statistically significant difference between NC-HepG2 group and HepG2 group. This suggested that the up-regulation of miR-101 expression inhibits HepG2 cell proliferation ability.

MiR-101 overexpression inhibits HepG2 cell migration and invasion abilities

Scratch test result (Fig. 3A) showed that the scratch healing rates in the miR-101-HepG2 group was significantly lower than the cells in the blank control group and negative control group $(P<0.05)$. The results indicated that upregulation of miR-101 
$\boldsymbol{A}$

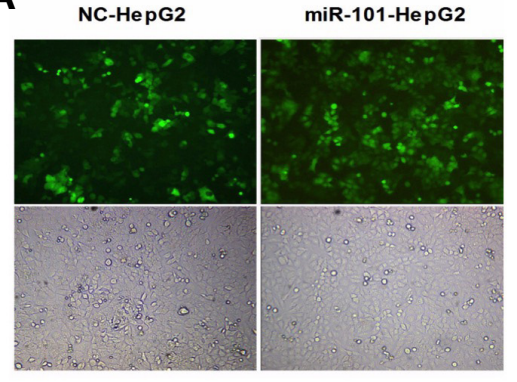

$B$

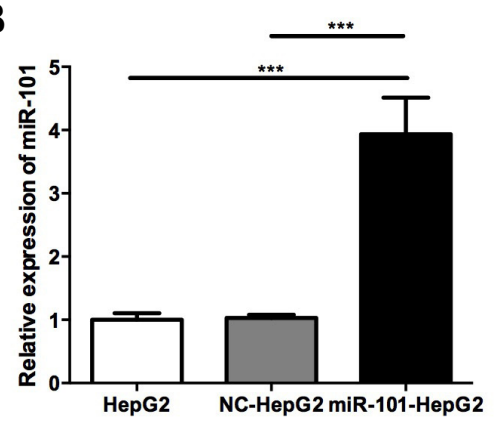

C

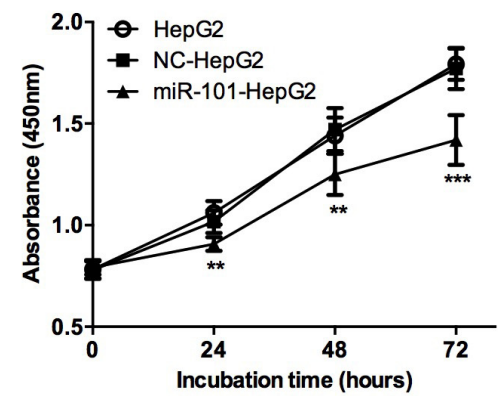

Fig. 2. Overexpression of miR-101 inhibited the proliferation ability of HepG2 cells. HepG2 cells were transfected with miR-101 mimics or NCmimics, and cells were divided into three groups: untreated HepG2 group, NC-HepG2 group and miR-101-HepG2 group. (A) Representative fluorescence microscopy picture $(\times 100$, the two small upper pictures $)$ and white light microscopy picture $(\times 100$, the two small lower pictures $)$ are shown in the lower left part. (B) RT-PCR method was used to detect the miR-101 expression levels in HepG2 group, the blank group and the negative control group after transfection of miR-101. The untreated group (HepG2 group) was set as 1. (C) CCK method was used to detect cell proliferation at specific time points, ${ }^{*} P<0.05,{ }^{*+*} P<0.001$

expression inhibits HepG2 cell migration.

The Transwell invasion test result (Fig. 3B) showed that the number of invading cells in overexpressing miR-101 group was much less than that in either the NC-treated groups or non-treated blank groups. These results suggested that upregulation of miR-101 expression inhibits invasion ability of HepG2 cells.

\section{Girdin is the target gene of miR-101 in HepG2 cells}

Girdin, which exhibits tumor promoter in many kinds of cancer. To test whether miR-101 inhibited tumor proliferation, migration and invasion through Girdin suppression we used western blot (Fig. 4A) and qRT-PCR (Fig. 4B) analyses to examine the expression of Girdin in HepG2 cells. In correspondence with our hypothesis, the results revealed that Girdin mRNA and protein expression levels were decreased when miR-101 was overexpressed.

To further explore this hypothesis, we amplified the Girdin 3'-UTR containing the target sequence (Supplementary Fig. S1) in which there are two possible biding sites (the underline position). Either left intact or mutated (Supplementary Fig. S2) sequence was inserted into a psiCHECK2 luciferase reporter vector. We detected fluorescence activity intensity of the dual luciferase reporter vectors, as shown in Fig. 4C. Luciferase activity intensity in cell samples co-transfected with WT-Girdin-3'-UTR-psi-CHECK2 plasmid and miR-101 mimics was significantly down-regulated than that in the other two groups (Fig. 4C). These results suggest that miR-101 directly combines to the 3'-UTR of Girdin and Girdin is a target gene of miR-101.
A

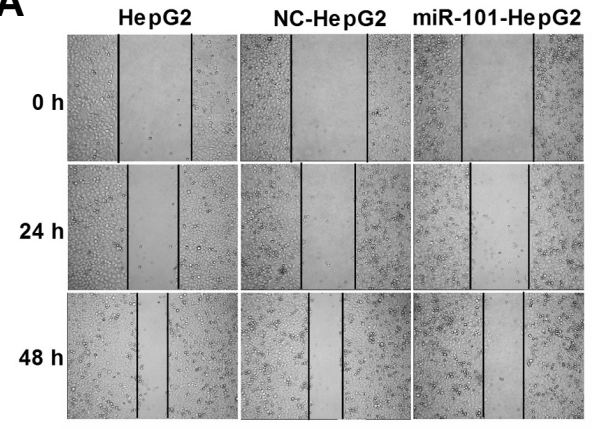

B

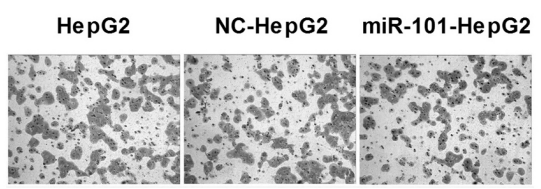

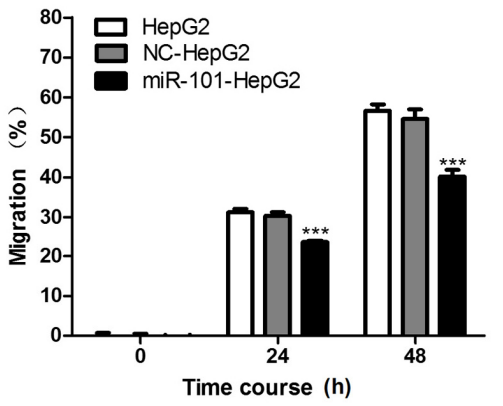

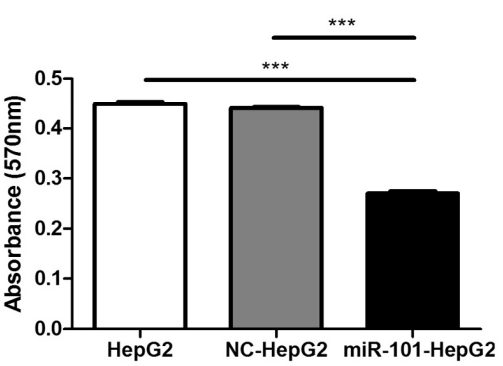

Fig. 3. Overexpression of $\mathrm{miR}-101$ inhibited HepG2 migration and invasion abilities. (A) The scratch test was used to detect cell migration rates at $0,24,48 \mathrm{~h}$, and the representative microscopy picture $(\times 40)$ was shown in the upper left part. (B) The Transwell test method was used to detect cell invasion ability. The representative microscopy picture $(\times 100)$ was shown in the lower left part, ${ }^{\text {tatk }} P<0.001$. 
A

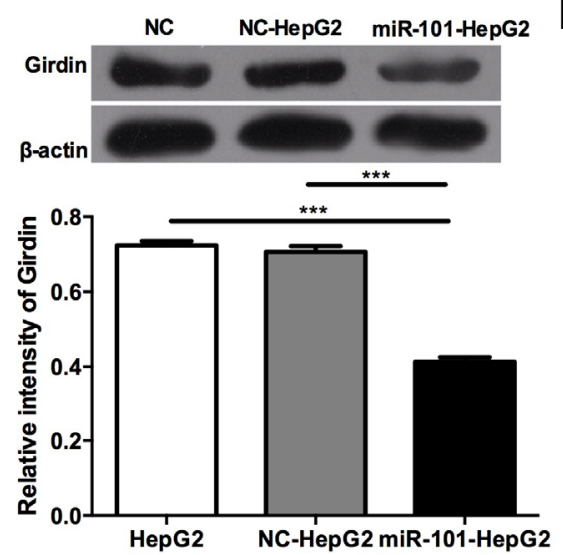

C

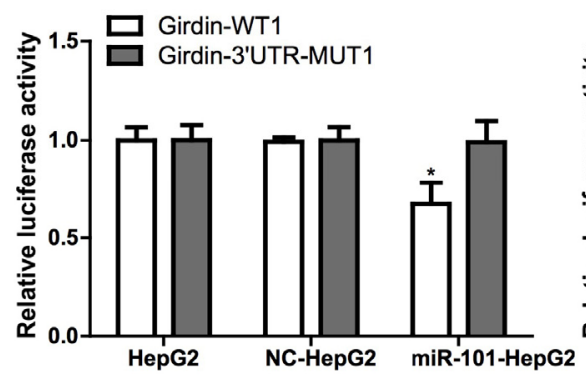

B
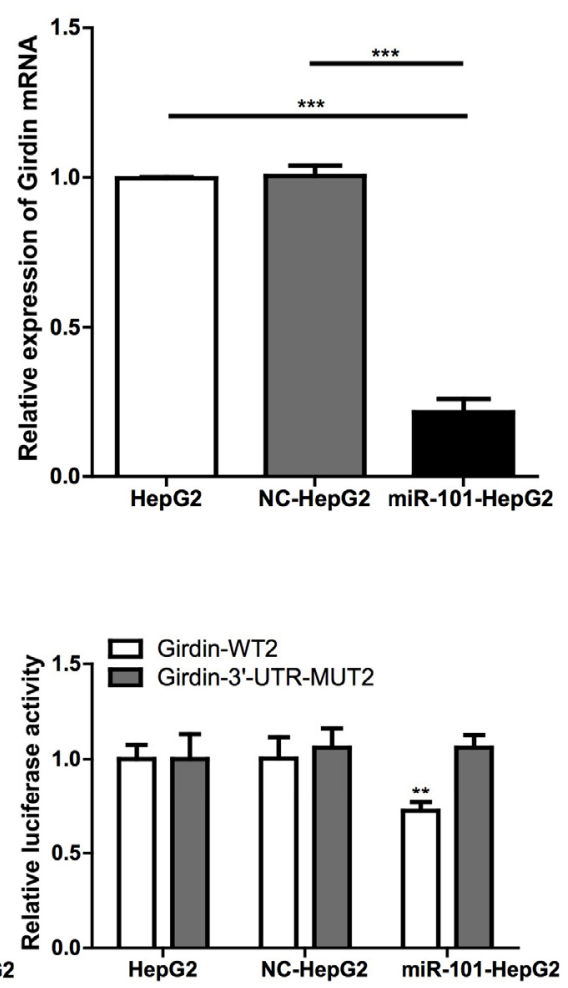

Fig. 4. Girdin is the target gene of miR-101. (A) Western blotting was used to detect the protein expression level of Girdin in HepG2 cells and $\beta$ actin was used as the internal control. (B) qRT-PCR was used to detect the expression levels of Girdin mRNA in different treatment groups. (C) Cells were divided into three groups: nontreated blank (HepG2), negative control (NC-HepG2), and hsa-mir-101 (miR-101-HepG2), and co-transfected with either the WT Girdin reporter vector (Girdin) or the mutant (GirdinMut) vector. Fluorescence activities in different groups were detected. The normalized luciferase activity in the control group was set to $1 .{ }^{*} P<0.05$, ${ }^{* *} P<0.01,{ }^{* * *} P<0.001$.

Fig. 5. Silencing Girdin expression inhibited proliferation ability of HepG2 cells. (A) The plasmid structure of Talen vector. (B) HepG2 cells were transfected plasmids in order to knockout of the Girdin gene. Forty-eight hours later Girdin expression levels were determined by western blotting. (C) CCK method was used to detect proliferation of HepG2 cell at indicated time points. ${ }^{\star} P<0.05,{ }^{,+*} P<$ 0.001 (NC vs. Girdin-KO), ${ }^{\#} P<0.01$, ${ }^{\#} P<0.001$ (Girdin-KO+miR-101 inhibitor vs. Girdin-KO).

B
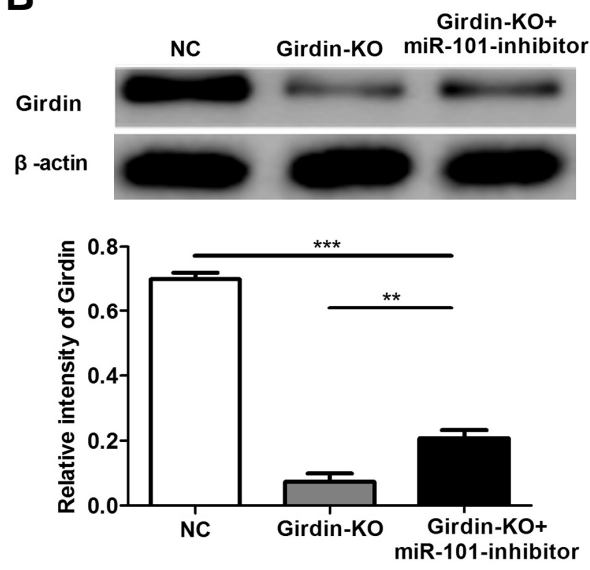

C

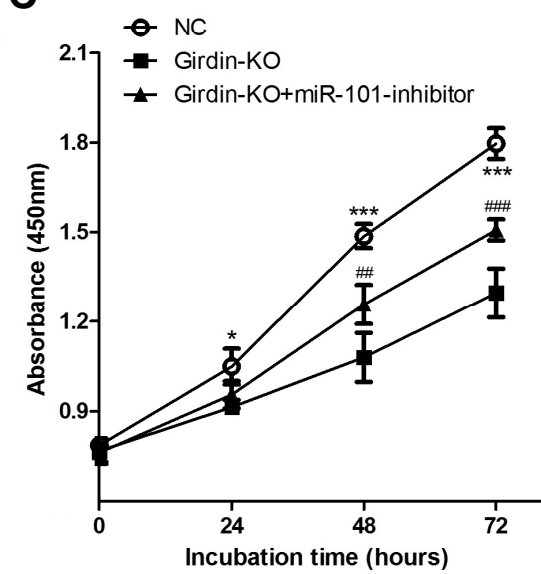


$\boldsymbol{A}$

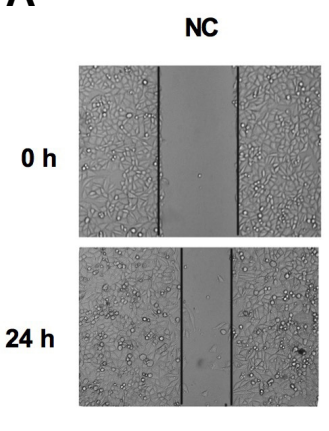

C

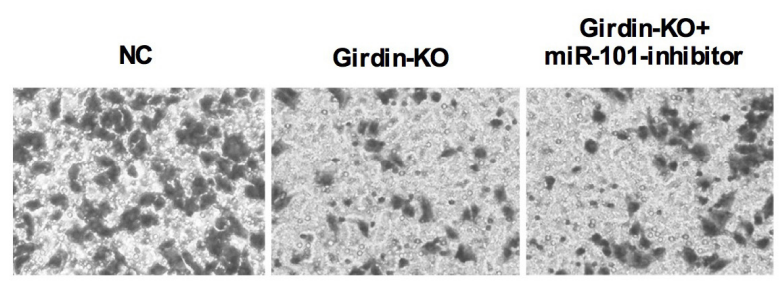

B

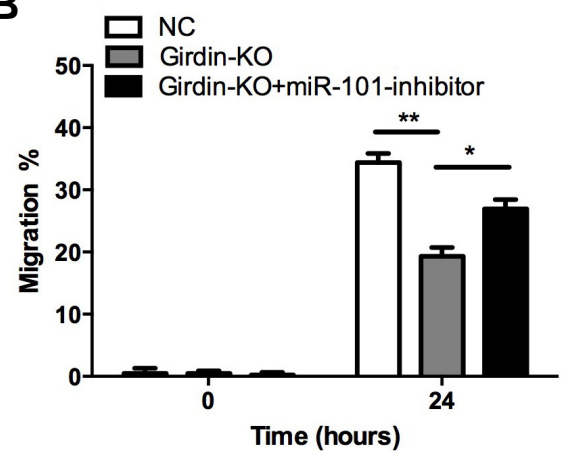

D

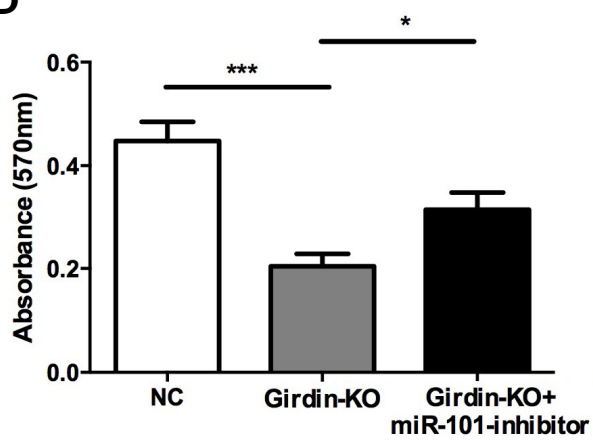

Fig. 6. Silencing Girdin expression inhibited migration and invasion abilities of HepG2 cells. (A, B) The scratch test was used to measure migration ability of HepG2 cell in different treatment groups. (C, D) The Transwell test was performed to detect cells invasion ability in different treatment groups. KO: knockout, ${ }^{*} P<0.05,{ }^{* *} P<0.01,{ }^{* t * *} P<0.001$.

\begin{abstract}
Silencing Girdin expression can inhibit proliferation and migration abilities of HepG2 cells

To further confirm the effects of miR-101/Girdin axis on HepG2 cells, the cells were divided into three groups, the group which was transfected with blank plasmid (NC), the group which was co-transfected with two Talen plasmids (Girdin-KO) and the group was con-transfected with two Talen plasmids and miR101 inhibitor (Girdin-KO+miR-101 inhibitor). Western blotting (Fig. 5B) were used to detect Girdin expression levels in HepG2 group (Girdin-KO) as well as the con-transfected group (Girdin-KO+miR-101 inhibitor) and the negative control group (NC). The results showed that Girdin had been successfully silenced in HepG2 cells while down-regulation of miR-101 significantly restored the Girdin expression level. CCK-8 assays (Fig. 5C), scratch (Figs. 6A and 6B) and transwell analysis (Figs. $6 \mathrm{C}$ and $6 \mathrm{D}$ ) were performed to test the proliferation, migration and invasion abilities of HepG2 cells in vitro. The results showed that knockout of Girdin expression also remarkably suppresses proliferation, migration and invasion abilities of HepG2 cells while down-regulation of miR-101 significantly restored the inhibitory effect, suggesting that miR-101 suppressed $\mathrm{HCC}$ development by mainly targeting Girdin.
\end{abstract}

\section{DISCUSSION}

In recent years, increasing evidence suggests that the dysregulation of miRNAs participates in many types of cancer and the abnormal expression of miR-101 is closely related to the progression of many tumors. Zhang et al. (2013) confirmed that miR-101 inhibits angiogenesis of cholangiocarcinoma by directly regulating the VEGF mRNA. Another study found that miR-
101 suppresses the proliferation of thyroid papillary cancer cells through targeting Rac1 (Lin et al., 2014). However, miR-101 also has been reported to increase pulmonary adenocarcinoma cell sensitivity to cisplatin by activating caspase 3-dependent apoptosis (Yin et al., 2014). The functions of miR-101 in HCC also have attracted an increasing amount of attention. Su et al. (2009) examined the expression levels of 308 kinds of miRNA in HCC tissue and found that miR-101 expression in HCC tissue was significantly lower. miR-101 exerts pro-apoptotic function through its target Mcl-1 in HCC (Su et al., 2009). However, the specific molecular mechanism of miR-101 in HCC remains to be elucidated.

In this study, miR-101 expression level in HCC tissue detected via qRT-PCR method. The results showed that the miR101 level in HCC tissue was significantly lower than that in the para-cancer tissue and miR-101 level in stage III/IV was lower compared with that in stage I/II. Meanwhile, Girdin mRNA expression level in HCC tissue was significantly higher in HCC tissues and Girdin level in stage III/IV was also higher than that in stage $\mathrm{I} / \mathrm{II}$. This result verified the results previously reported in the literature. The correlation analysis was showed that a negative correlation between miR-101 and Girdin expression levels.

To further explore the effect of miR-101 in vitro, we overexpressed miR-101 in HepG2 cells. The cell proliferation assays revealed that miR-101 suppresses proliferation of HepG2 cells. The scratch and Transwell analysis revealed that miR-101 inhibits cell migration and invasion abilities. These results demonstrated that up-regulation of miR-101 expression can effectively inhibit the proliferation, migration and invasion abilities of HepG2 cells. The aforementioned results were consistent with the previously study results (Shen et al., 2014; Su et al., 2009). 
However, the relationship between miR-101 and Girdin has not been reported. To further verify whether Girdin is the target gene of miR-101, qRT-PCR and western blot analyses were performed to detect the expression of Girdin in HepG2 cells. We found that Girdin mRNA and its protein expression levels were significantly decreased after miR-101 mimics were transfected into the HepG2 cell. The dual luciferase gene analysis confirmed that miR-101 could directly bind to Girdin mRNA 3'UTR. Therefore, Girdin is the target gene of miR-101 in HCC.

In this study, Tanlen technology was used to perform the knockout of Girdin. After co-transfected with Talen plasmids and miR-101 inhibitor, CCK-8 test , scratch test and Transwell test were used to further verify tumor promotion effect of Girdin. The results showed that after knockout of Girdin limited proliferation, migration and invasion abilities of HepG2 cell, yet downregulation miR-101 significantly recovered this inhibitory effect, suggesting that miR-101 suppressed HCC development by mainly targeting Girdin.

In summary, our study demonstrated that miR-101 expression is dramatically decreased in HCC tissues compared with that in matched normal tissues. Upregulation of miR-101 remarkably inhibits HepG2 cell proliferation, migration and invasion abilities. As a target gene of miR-101, knockout of Girdin also suppressed HepG2 cell proliferation, migration and invasion while down-regulation of miR-101 significantly restored the inhibitory effect. Therefore, the miR-101/Girdin pathway may provide a new direction for the clinical treatment of HCC.

Note: Supplementary information is available on the Molecules and Cells website (www.molcells.org).

\section{ACKNOWLEDGMENTS}

This work was supported by National Natural Science Foundation of China (Grant No. 81572965, 81372140, 81301688, 81272192, 81171882), Ph.D. Programs Foundation of Ministry of Education of China (No. 20130162110050 and 20130162120093), Program for New Century Excellent Talents in University (NCET-11-0527), China Postdoctoral Science Foundation (2014M552167), Post-doctoral Foundation of Central South University (No. 131425), Project of the Department of Science and Technology of Hunan Province (No. 2013FJ6003), Fundamental Research Funds for the Central Universities of Central South University (2015zzts277), 125 Talent Project of the Third Xiangya Hospital of Central South University.

\section{REFERENCES}

Anai, M., Shojima, N., Katagiri, H., Ogihara, T., Sakoda, H., Onishi, Y., Ono H., Fujishiro, M., Fukushima, Y., Horike, N., et al. (2005). A novel protein kinase $b$ (pkb)/akt-binding protein enhances $\mathrm{pkb}$ kinase activity and regulates DNA synthesis. J. Biol. Chem. 280, 18525-18535.

Cao, K., Jiang, W., Cao, P., Zou, Q., Xiao, S., Zhou, J., and Huang, C. (2014). Talen-mediated girdin knockout downregulates cell proliferation, migration and invasion in human esophageal carcinoma eca109 cells. Mol. Med. Rep. 10, 848-854.

Cao, K., Lu, C., Han, S., Zou, Q., Li, J., Xie, D., He, S., Yu, L., Zhou, J., Peng, X., et al. (2015). Expression of Girdin in primary hepatocellular carcinoma and its effect on cell proliferation and invasion. Int. J. Clin. Exp. Pathol. 8, 551-559.

Enomoto, A., Murakami, H., Asai, N., Morone, N., Watanabe, T., Kawai, K., Murakumo, Y., Usukura, J., Kaibuchi, K., and Takahashi, M. (2005). Akt/pkb regulates actin organization and cell motility via girdin/ape. Dev. Cell. 9, 389-402.
Jin, F., Liu, C., Guo, Y., Chen, H., and Wu, Y. (2013). Clinical implications of Girdin and PI3K protein expression in breast cancer. Oncol Lett. 5,1549-1553.

Kim, K., Kim, J.H., Kim, I., Lee, J., Seong, S., Park, Y.W., and Kim, N. (2015). MicroRNA-26a regulates RANKL-induced osteoclast formation. Mol. Cells 38, 75-80.

Landgraf, P., Rusu, M., Sheridan, R., Sewer, A., lovino, N., Aravin, A., Pfeffer, S., Rice, A., Kamphorst, A.O., Landthaler, M., et al (2007). A mammalian microrna expression atlas based on small RNA library sequencing. Cell 129, 1401-1414.

Le-Niculescu, H., Niesman, I., Fischer, T., DeVries, L., and Farquhar M.G. (2005). Identification and characterization of GIV, a novel Galpha i/s-interacting protein found on COPI, endoplasmic reticulum-Golgi transport vesicles. J. Biol. Chem. 280, 2201222020.

Lin, X., Guan, H., Li, H., Liu, J., Wei, G., Huang, Z., Liao, Z., and Li, Y. (2014). MiR-101 inhibits cell proliferation by targeting rac1 in papillary thyroid carcinoma. Biomed. Rep. 2, 122-126.

Peng, X., Cao, P., Li, J., He, D., Han, S., Zhou, J., Tan, G., Li, W., Yu F., Yu, J., et al. (2015). MiR-1204 sensitizes nasopharyngeal carcinoma cells to paclitaxel both in vitro and in vivo. Cancer Biol Ther 16, 261-267.

Siegel, R., Naishadham, D., and Jemal A. (2012). Cancer statistics, 2013. CA. Cancer. J. Clin. 62,10-29.

Shen, Q., Bae, H.J., Eun, J.W., Kim, H.S., Park, S.J., Shin, W.C., Lee, E.K., Park, S., Park, W.S., Lee, J.Y., et al. (2014). MiR-101 functions as a tumor suppressor by directly targeting nemo-like kinase in liver cancer. Cancer. Lett. 344, 204-211.

Shibata, T., Matsuo, Y., Shamoto, T., Hirokawa, T., Tsuboi, K., Takahashi, H., Ishiguro, H., Kimura, M., Takeyama, H., and Inagaki, H. (2013). Girdin, a regulator of cell motility, is a potential prognostic marker for esophageal squamous cell carcinoma. Oncol. Rep. 29, 2127-2132.

Simpson, F., Martin, S., Evans, TM., Kerr, M., James, D.E., Parton, R.G., Teasdale, R.D., and Wicking, C. (2005). A novel hookrelated protein family and the characterization of hook-related protein 1. Traffic 6. 442-458.

Su, H., Yang, J.R., Xu, T., Huang, J., Xu, L., Yuan, Y., and Zhuang, S.M. (2009). MicroRNA-101, down-regulated in hepatocellular carcinoma, promotes apoptosis and suppresses tumorigenicity. Cancer Res. 69, 1135-1142.

Tameda, M., Sugimoto, K., Shiraki, K., Yamamoto, N., Okamoto, R., Usui, M., Ito, M., Takei, Y., Nobori, T., Kojima, T., et al. (2014) Collagen triple helix repeat containing 1 is overexpressed in hepatocellular carcinoma and promotes cell proliferation and motility. Int. J. Oncol. 45, 541-548.

Wang, C., Lin, J., Li, L., and Wang, Y. (2014). Expression and clinical significance of girdin in gastric cancer. Mol. Clin. Oncol. 2 425-428.

Xu, X., Chen, H., Lin, Y., Hu, Z., Mao, Y., Wu, J., Xu, X., Zhu, Y., Li, S., Zheng, X., et al. (2013). MicroRNA-409-3p inhibits migration and invasion of bladder cancer cells via targeting c-Met. Mol. Cells. 36, 62-68.

Yin, J., Wang, M., Jin, C., and Qi, Q. (2014). miR-101 sensitizes A549 NSCLC cell line to CDDP by activating caspase 3dependent apoptosis. Oncol. Lett. 7, 461-465.

Zhang, J., Han, C., Zhu, H., Song, K., and Wu, T. (2013). MiR-101 inhibits cholangiocarcinoma angiogenesis through targeting vascular endothelial growth factor (VEGF). Am. J. Pathol. 182, 1629-1639.

Zhang, Y.J., Li, A.J., Han, Y., Yin, L., and Lin, M.B. (2014). Inhibition of Girdin enhances chemosensitivity of colorectal cancer cells to oxaliplatin. World J Gastroenterol. 20, 8229-8236.

Zheng, C., Li, J., Wang, Q., Liu, W., Zhou, J., Liu, R., Zeng, Q., Peng, X., Huang, C., Cao P., et al. (2015). MicroRNA-195 functions as a tumor suppressor by inhibiting CBX4 in hepatocellular carcinoma. Oncol. Rep. 33, 1115-1122.

Zhou, L., Yang, Z.X., Song, WJ., Li, QJ., Yang, F., Wang, D.S., Zhang, N., and Dou, K.F. (2013). Microrna-21 regulates the migration and invasion of a stem-like population in hepatocellular carcinoma. Int. J. Oncol. 43, 661-669. 